\title{
Base Line Wander, Breathing, Power Line Interference Noise Suppression and QRS Detection in Scilab
}

\author{
Imteyaz Ahmad \\ Associate Professor \\ ECE Department, BIT Sindri
}

\author{
Amar Prakash Sinha \\ Associate Professor \\ ECE Department, BIT Sindri
}

\begin{abstract}
The ECG signal contain base line wander noise $(0.5$ to $0.6 \mathrm{~Hz})$, breathing noise $(5 \mathrm{~Hz})$, power line interference $(50 \mathrm{~Hz})$ and EMG noise(above $100 \mathrm{~Hz}$ ).Electrode Motion artifact noise can be reduced by minimizing movement made by the patient. High pass filter can be used to remove base line wander noise with cutoff frequency of $0.6 \mathrm{~Hz}$. High pass filter can be used to remove breathing noise with cutoff frequency of $6 \mathrm{~Hz} .50$ $\mathrm{Hz}$ power line interference can be removed using band stop filter. QRS detection is done using differentiation method. Scilab. is used for performing signal processing task of removing common noise in ECG signal. SNR of Input signals with base line wander noise, breathing and PLI noise when passed to FIR(high pass and band stop filter order 51) shows improvement in output SNR.
\end{abstract}

\section{Keywords}

Base line wander noise, breathing noise, power line interference, QRS detection, Scilab

\section{INTRODUCTION}

The ECG may be monitored continuously when the patient is in emergency care, in a coronary care unit, an intensive care unit or during stress tests[1]. In these cases only one lead, usually lead II is monitored, on a display[2]. ECG machine is a vital part of hospitals and aid the doctors to keep track of patient's vital sign during emergency. The electrocardiogram is the graphic recording or display of time variant voltage produced by the myocardium during Cardiac cycle. The electrocardiogram is used clinically in diagnosing various diseases and conditions associated with the heart[3]. It also serves as a timing reference for other measurements. Engineers working in the medical profession are encouraged to learn as much as possible about medical and hospital practices and in particular about physiology of human body[4]. It is only by gaining such an understanding that they can communicate intelligently with medical professionals. This interaction between the two fields has led to the development of sophisticated medical equipment and systems. The monitoring mode bandwidth is $0.5-50 \mathrm{~Hz}$ as only rhythmic information is required. The tracing of voltage difference at any two sites due to the electrical activity of the heart is called a lead. Although two electrodes can be attached to any part of the body to lead the heart current to the galvanometer, it is customary to make use of the forearms, the left leg and the pericardium. Each chamber of the heart produces a characteristics electrocardiographic pattern. Since the electrical potentials over the various areas of the heart differ, the recorded tracing from each limb vary accordingly The heart is made of a special kind of muscle, so that it can beat automatically without having to be told to do so by the brain. The left side of the heart drives oxygen rich blood out of the aortic semi-lunar outlet valve into circulation where it is delivered to all parts of the body. Blood returns to the right side of the heart low in oxygen and high in carbon dioxide and is then pumped through the pulmonary semi-lunar pulmonic valve to the lungs to have its oxygen supply replenished before returning to the left side of the heart to begin the cycle again. We have to suppress $50 \mathrm{~Hz}$ noise, motion artifact, EMG noise and baseline wander. We have used derivative based algorithms for QRS detection. To remove baseline wander and power-line interference from the ECG, a bandpass filter is applied, while to enhance the QRS complex, a five-point first-order differentiation, absolute and backward cumulation operation were applied.

\section{COMMON ECG NOISES}

\subsection{Baseline Wander}

Baseline wander or baseline drift is the effect where the base axis (x-axis) of a signal appears to 'wander' or move up and down rather than be straight. This causes the entire signal to shift from its normal base. In ECG signal, the baseline wander is caused due to improper electrodes electrode-skin impedance), patient's movement and breathing (respiration).

\subsection{Power Line Interference}

Electromagnetic fields caused by a power line represent a common noise source in the ECG, as well as to any other bioelectrical signal recorded from the body surface. Such noise is characterized by 50 or $60 \mathrm{~Hz}$ sinusoidal interference, possibly accompanied by a number of harmonics. Such narrowband noise renders the analysis and interpretation of the ECG more difficult, since the delineation of lowamplitude waveforms becomes unreliable and spurious waveforms may be introduced. It is necessary to remove power line interference from ECG signals as it completely superimposes the low frequency ECG waves like $\mathrm{P}$ wave and T wave.

\section{METHOD}

ECG signal is taken from physionet database. ECG measurements may be corrupted by many sorts of noise. The ones of primary interest are: Power line interference, base line wander noise. These artifacts strongly affects the ST segment, degrades the signal quality, frequency resolution, produces large amplitude signals in ECG that can resemble PQRST waveforms and masks tiny features that are important for clinical monitoring and diagnosis. Cancelation of these artifacts in ECG signals is an important task for better diagnosis[5].

\section{ECG SIGNAL IMPORT IN SCILAB}

In Scilab Microsoft Office Excel i.e. Excel 97-2003 Worksheet (.xlsx) file can be accessed directly into the workspace. A variable can be assigned to ECG amplitude.[6] 


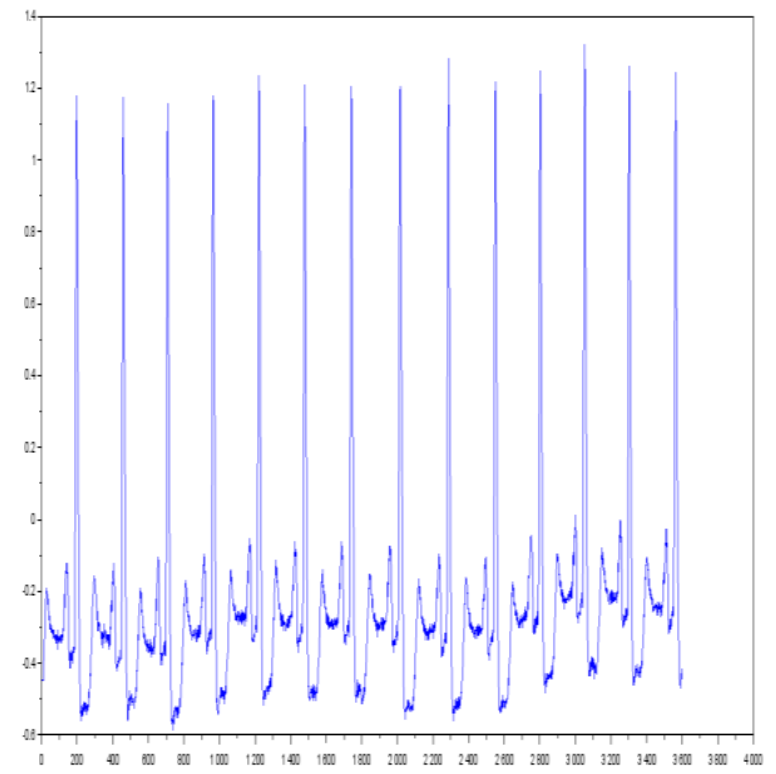

Figure 1: ECG signal MITBIH105 from Physionet

\section{BASE LINE WANDER NOISE ELIMINATION}

Base line wander noise[7] of frequency $0.5 \mathrm{~Hz}$ can be added to above ECG. High pass filter with cutoff frequency of 2 $\mathrm{Hz}$ can be used. Input SNR is $-1.6922 \mathrm{~dB}$. Result is shown below in Figure 2.SNR of output signal is $-1.4963 \mathrm{~dB}$.

Codes: $\mathbf{f s}=\mathbf{3 6 0}$;

$\mathrm{t}=\mathbf{0}: \mathbf{1} / \mathbf{f s}: \mathbf{1 0}-\mathbf{1} / \mathbf{f s}$

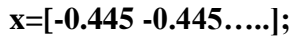

$\mathrm{n}=\sin (\% \mathrm{pi} * \mathrm{t} / \mathbf{1 0})$;

$\mathbf{y}=\mathbf{x}+\mathbf{n}$;

fc $=2$;

$\mathbf{F c n}=2 * \mathbf{f c} / \mathbf{f s}$;

h=wfir("hp",51,[Fcn],"re",[Fcn]);

$\mathbf{r}=\mathbf{f i l t e r}(\mathbf{h}, \mathbf{1 , y})$;

clf;

subplot $(5,1,1)$;

$\operatorname{plot}(\mathbf{x})$;

subplot $(5,1,2)$;

$\operatorname{plot}(\mathbf{n})$;

$\underline{\text { subplot }}(\mathbf{5 , 1 , 3 )}$;

$\operatorname{plot}(y)$;

subplot $(5,1,4)$;

plot(r);
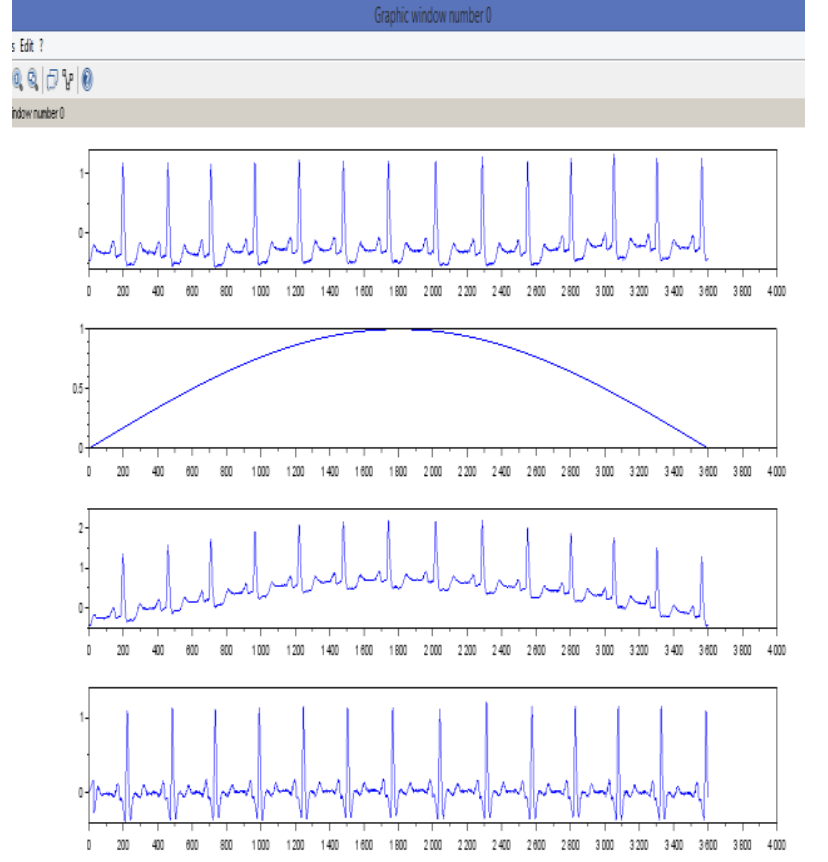

Figure 2: $1^{\text {st }}$ plot is pure ECG, $2^{\text {nd }}$ plot is base line wander noise, $3^{\text {rd }}$ plot is ECG with BLW noise and $4^{\text {th }}$ plot is the filtered ECG

\section{ELIMINATION OF BREATHING NOISE OF 5HZ IN ECG USING SCILAB}

The input SNR is $-5.3086 \mathrm{~dB}$. The output SNR is $-6.5595 \mathrm{~dB}$.

fs $=360$;

$\mathrm{t}=0: 1 / \mathrm{fs}: 10-1 / \mathbf{f s}$

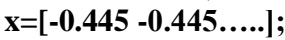

$\mathrm{n}=\sin (\% \mathrm{pi} * \mathrm{t})$;

$\mathbf{y}=\mathbf{x}+\mathbf{n}$;

fc $=6$;

Fen $=2 * \mathrm{fc} / \mathrm{fs}$;

h=wfir("hp",51,[Fcn],"re",[Fcn]);

$\mathbf{r}=$ filter $(\mathbf{h}, \mathbf{1}, \mathbf{y})$;

clf;

subplot $(5,1,1)$

$\operatorname{plot}(x)$;

subplot $(5,1,2)$;

plot(n);

$\underline{\text { subplot }}(5,1,3)$;

$\operatorname{plot}(y)$;

$\underline{\text { subplot }}(5,1,4)$;

plot(r); 
10
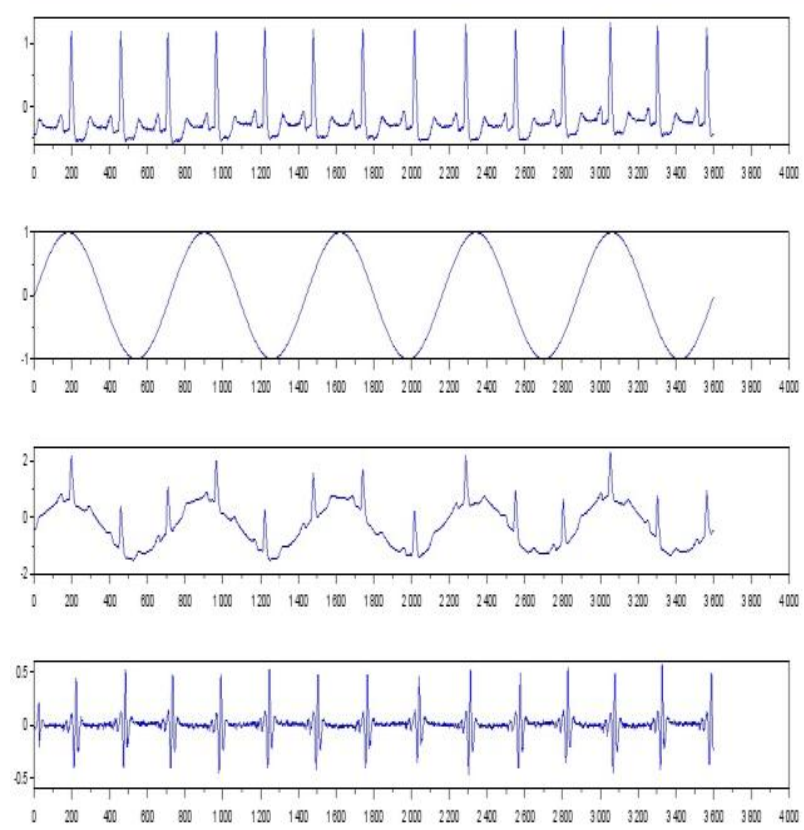

Figure 3: $1^{\text {st }}$ plot is pure ECG, $2^{\text {nd }}$ plot is breathing noise, $3^{\text {rd }}$ plot is ECG with Breathing noise and $4^{\text {th }}$ plot is the filtered ECG

\section{ELIMINATION OF PLI NOISE IN ECG USING SCILAB}

The input SNR is $-5.3086 \mathrm{~dB}$. The output SNR is $-1.1451 \mathrm{~dB}$.

fs $=360$;

$\mathrm{t}=\mathbf{0}: \mathbf{1} / \mathbf{f s}: 10-1 / \mathrm{fs}$

$\mathrm{x}=[-\mathbf{0 . 4 4 5}-\mathbf{- 0 . 4 4 5} \ldots . .$.$] ;$

$\mathrm{n}=\sin (100 * \% \mathrm{pi} * \mathrm{t})$;

$\mathbf{y}=\mathbf{x}+\mathbf{n}$;

fcl=20;

fch $=80$;

$\mathrm{fcn}=[2 * \mathrm{fcl} / \mathrm{fs} 2 * \mathrm{fch} / \mathrm{fs}]$

h=wfir("sb",51,[fcn],"re",[fcn]);

$\mathbf{r}=$ filter $(\mathbf{h}, \mathbf{1}, \mathbf{y})$;

figure;

subplot $(4,1,1)$;

$\operatorname{plot}(\mathbf{t}, \mathbf{x})$;

subplot $(4,1,2)$;

plot $(\mathbf{t}, \mathbf{n})$;

subplot $(4,1,3)$;

plot $(t, y)$

subplot $(4,1,4)$;

$\underline{\operatorname{plot}}(\mathbf{t}, \mathbf{r})$

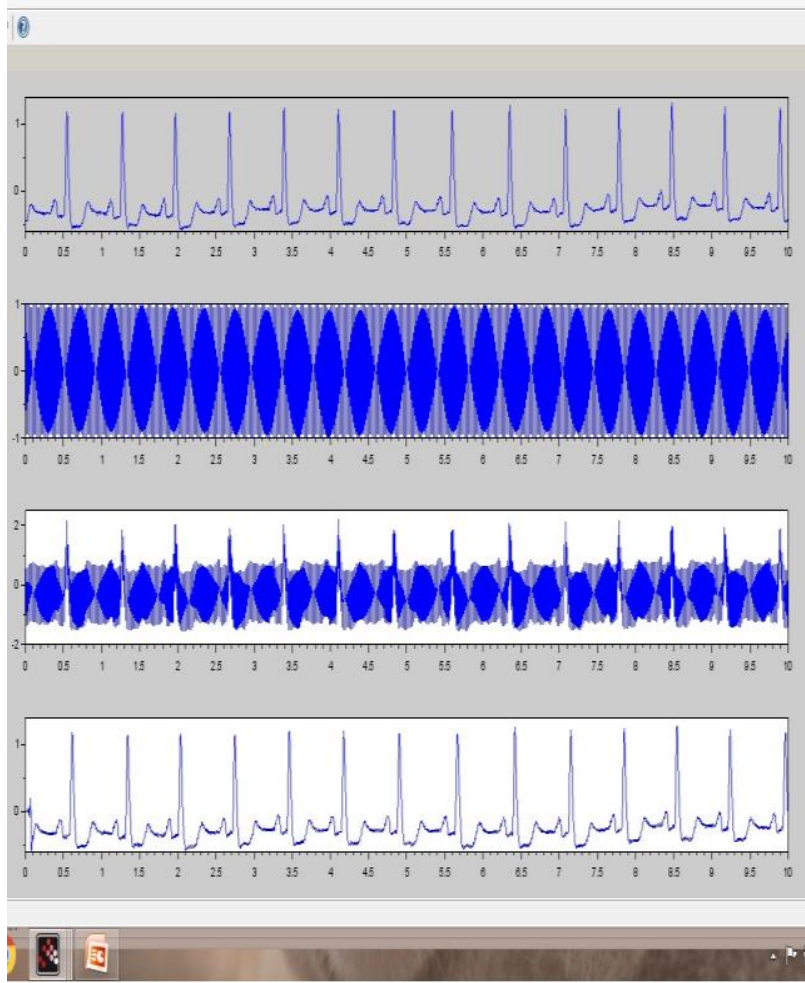

Figure 4: $1^{\text {st }}$ plot is pure ECG, $2^{\text {nd }}$ plot is PLI noise, $3^{\text {rd }}$ plot is ECG with PLI noise and $4^{\text {th }}$ plot is the filtered ECG

\section{QRS DETECTION BY DERIVATIVE BASED METHOD USING SCILAB}

Derivative operator enhances the QRS Complex and suppress $\mathrm{P}$ and $\mathrm{T}$ wave. $[8,9,10,11,12]$

fs $=360$;

$\mathrm{N}=0: 1: 3599$;

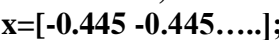

$\mathrm{x}=\mathrm{x}^{\prime}$;

h=wfir("bp",51,[5/180 15/180],"re",[5/180 15/180]);

$\mathbf{y}=$ filter $(\mathbf{h}, 1, \mathbf{x})$;

h1 $=[1,0,-1,0,0]$;

y1=filter(h1,1,y);

h1sm $=[.25, .5, .25,0,0]$;

y1sm=filter(h1sm,1,y1);

figure; plot(n,x);

figure;

plot $(\mathbf{n}, \mathbf{y})$;

figure;

$\operatorname{plot}(\mathbf{n}, \mathbf{y 1})$;

figure;

plot(n,y1sm);

title('y1sm');

xlabel('no. of sample');

ylabel('amplitude in $\mathbf{m V}$ ');

h2 $=[1,0,-2,0,1]$;

y2=filter(h2,1,y1sm);

h2sm $=[.25, .5, .25,0,0]$;

y2sm=filter(h2sm,1,y2);

figure;

plot $(\mathbf{n}, \mathbf{y} 2)$;

figure;

plot(n,y2sm);

title ('y2sm');

xlabel('no. of sample');

ylabel ('amplitude in $\mathbf{m V}$ '); 
h3sm=1.3. *h1sm+1.1.*h2sm;

y3=filter(h3sm,1,x);

y3sm $=y 3$;

figure;

plot(n,y3sm);

title('y3sm');

xlabel('no. of sample');

ylabel ('amplitude in $\mathrm{mV}^{\prime}$ );

$\mathrm{N}=\mathbf{4 0}$;

h4=ones $(\mathrm{N}, 1) / \mathrm{N}$;

y4=filter(h4,1,y3sm);

y4sm=y4;

$\mathrm{y} 4 \mathrm{sm}=\mathrm{y} 4 \mathrm{sm}+1.2$;

figure;

plot(n,y4sm);

title('y4sm');

xlabel('no. of sample');

ylabel('amplitude in $\mathbf{m V}$ ');

subplot $(7,1,1)$; $\operatorname{plot}(\mathbf{n}, \mathbf{x})$;

title('input ECG');

subplot $(7,1,2)$

plot $(\mathbf{n}, \mathbf{y})$;

title('output of bandpass filter5-15'); subplot $(7,1,3)$;

plot $(\mathbf{n}, \mathbf{y} 1 \mathrm{sm})$;

title('y1sm');

title('output of first derivative operator'); $\underline{\operatorname{subplot}}(7,1,4)$; plot $(\mathbf{n}, \mathbf{y} 2 \mathrm{sm})$;

$\underline{\text { title }}($ 'y2sm');

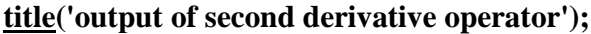

subplot $(7,1,5)$

plot $(\mathbf{n}, \mathbf{y} 3 \mathrm{sm})$;

title('y3sm');

title $(' y 3 \mathrm{sm}=1.3 . * \mathrm{y} 1 \mathrm{sm}+1.1$. $* \mathrm{y} 2 \mathrm{sm}$ combine result'); subplot $(7,1,6)$

plot(n,y4sm);

title('passing y3sm to moving average filter in QRS duration for smooth single pulse');
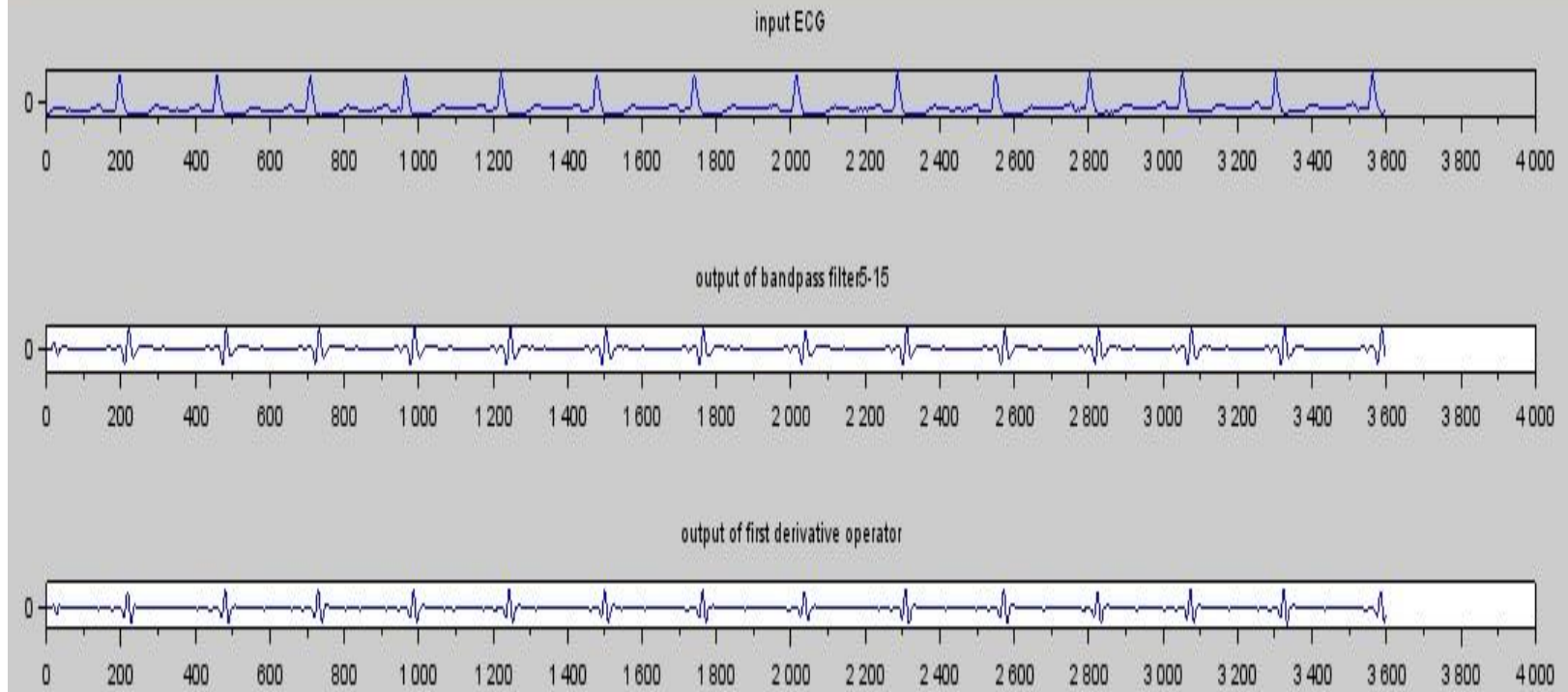

output of second derivative operator

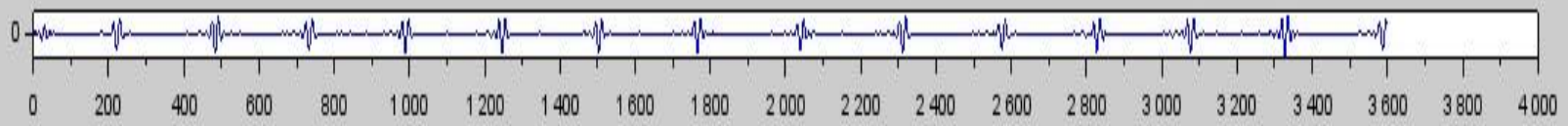

Y3sm=1.3.'y1sm+1.1.'Y2sm combine lesult

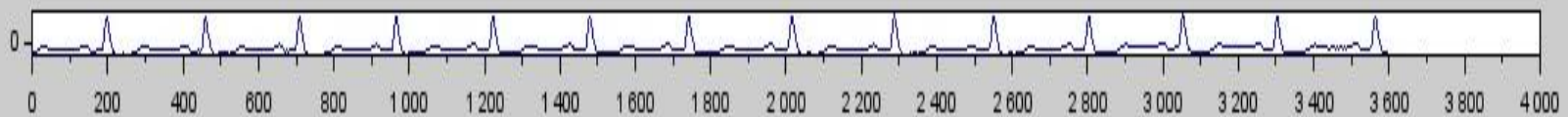

passing y Bsm to moving average filter in QRS duration for smooth single pulse

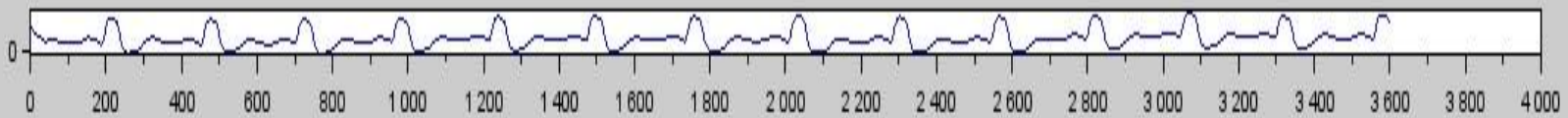


Figure 5: $1^{\text {st }}$ plot is pure ECG, $2^{\text {nd }}$ plot is output of bandpass filter, $3^{\text {rd }}$ plot is output of first derivative and $4^{\text {th }}$ plot is the output of second derivative, $5^{\text {th }}$ plot is combined output of first and second derivative, $6^{\text {th }}$ plot is the output of moving average filter for single pulses of QRS duration.FIR filter

\section{OBSERVATION AND RESULT}

Base line wander noise $(0.5 \mathrm{~Hz})$ as seen in Figure 2 can be eliminated using FIR high pass filter order 51 and cutoff frequency of $2 \mathrm{~Hz}$. Breathing noise $(5 \mathrm{~Hz})$ as seen in Figure 3 can be eliminated using FIR high pass filter order 51 and cutoff frequency of $6 \mathrm{~Hz}$. PLI noise $(50 \mathrm{~Hz})$ as seen in Figure 4 can be eliminated using FIR band stop filter order 51 and $\mathrm{Flc}=20 \mathrm{~Hz}$ and $\mathrm{Fhc}=80$ Hz.QRS detection is done using differentiation method. Band pass filter is having band width of $5-15 \mathrm{~Hz}$ for QRS detection.FIR high pass filter order of 5 are used for first and second derivative. The combined output shows QRS pulses. These are smoothed using moving average filter order 40 for $\mathrm{Fs}=360$. The output of moving average filter(low pass) consists of single pulses of QRS duration.

\begin{tabular}{|l|l|l|l|}
\hline $\begin{array}{l}\text { Input signal } \\
\text { with: }\end{array}$ & Input SNR & Output SNR & $\begin{array}{l}\text { Mean } \\
\text { square error }\end{array}$ \\
\hline $\begin{array}{l}\text { Base line } \\
\text { wander noise }\end{array}$ & $-1.6922 \mathrm{~dB}$ & $-1.4963 \mathrm{~dB}$. & $1.3754 \mathrm{e}-04$ \\
\hline $\begin{array}{l}\text { Breathing } \\
\text { noise }\end{array}$ & $-5.3086 \mathrm{~dB}$ & $-6.5595 \mathrm{~dB}$. & $4.5850 \mathrm{e}-05$ \\
\hline PLI noise & $-5.3086 \mathrm{~dB}$ & $-1.1451 \mathrm{~dB}$. & $0.51 \mathrm{e}-6$ \\
\hline
\end{tabular}

\section{CONCLUSION}

Common noise in ECG like base line wander, breathing and PLI noises are eliminated with high pass and band stop filter. Signal processing is done using Scilab.QRS detection is performed using differentiation method. Differentiation is basically high pass filtering and moving averaging is low pass filtering. Single QRS pulses are obtained after moving average filtering with order of 40.From the result it is clearly proved that SNR and MSE of the output signal is much improved than the input signal.

\section{REFERENCES}

[1] Sattar Y, Chhabra L. Electrocardiogram. [Updated 2020 Jul 31]. In: StatPearls [Internet]. Treasure Island (FL): StatPearls Publishing; 2020 Jan-. Available from: https://www.ncbi.nlm.nih.gov/books/NBK549803/

[2] Francis J. ,ECG monitoring leads and special leads, Indian Pacing and Electrophysiology journal, Issue-3 ,Volume-16, 2016
[3] Zègre-Hemsey, Jessica K et al. "Cardiac Monitoring in the Emergency Department." Critical care nursing clinics of North America vol. 28,3 (2016): 331-45. doi:10.1016/j.cnc.2016.04.009

[4] Institute of Medicine (US) Division of Health Sciences Policy. Medical Education and Societal Needs: A Planning Report for the Health Professions. Washington (DC): National Academies Press (US); 1983. Chapter 4, THE PHYSICIAN'S ROLE IN A CHANGING HEALTH CARE SYSTEM. Available from: https://www.ncbi.nlm.nih.gov/books/NBK217690/

[5] K Manivel, R samson Ravindran"Noise removal for baseline wander and Power line in ECG signals", IJAREEIE, Issue-2, Volume-4, 2015

[6] T. Aarthi, G. Sasi, P. Subapradha1, B. Subathra Thangam1, Ch. Sai Sita Sri, V. Elamaran1" A tutorial on digital filtering using discrete fourier transform with scilab" ,International Journal of Pure and Applied Mathematics, Special Issue on mathematical modeling of Engineering problems, Issue-15, Vol.-119,2018

[7] De Pinto V,"Filters for the reduction of baseline wander and muscle artifact in the ECG", J Electrocardiol. 1992; 25 Suppl: 40-8.

[8] Pan, J.; Tompking, W.J. A real-time QRS detection algorithm. IEEE Trans. Biomed. Eng. 1985, 32, 230 236.[CrossRef] [PubMed]

[9] Hamilton, P.S.; Tompkins, W.J. Quantitative investigation of $\mathrm{QRS}$ detection rules using MIT/BIH arrhythmia database. IEEE Trans. Biomed. Eng. 1986, 12, 1157-1165. [CrossRef]

[10] He, R.; Wang, K.; Li, Q.; Yuan, Y.; Zhao, N.; Liu, Y.; Zhang, $\mathrm{H}$. A novel method for the detection of R-peaks in ECG based on K-Nearest Neighbors and Particle Swarm Optimization. EURASIP J. Adv. Signal Process. 2017,2017. [CrossRef]

[11] Lastre-Domínguez C, Shmaliy YS, Ibarra-Manzano O, Munoz-Minjares J, Morales-Mendoza LJ. ECG Signal Denoising and Features Extraction Using Unbiased FIR Smoothing. Biomed Res Int. 2019;2019:2608547. Published 2019 Feb 20. doi:10.1155/2019/2608547.

[12] Christov II, Daskalov IK. Filtering of electromyogram artifacts from the electrocardiogram. Med Eng Phys. 1999 Dec; 21(10):731-6. doi: 10.1016/s13504533(99)00098-3. PMID: 10717553 